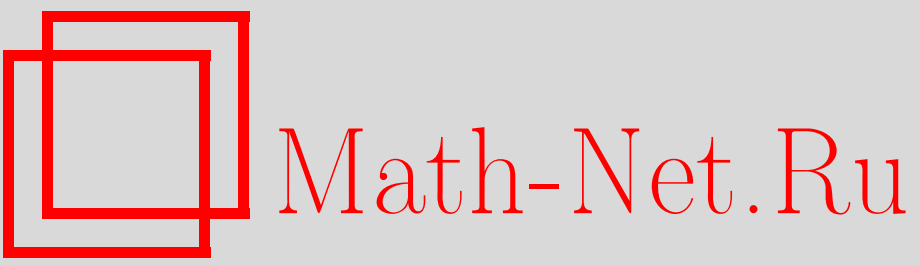

М. В. Шамолин, Определение относительной грубости и двупараметрическое семейство фазовых портретов в динамике твердого тела, УМH, 1996, том 51, выпуск 1, 175-176

DOI: https://doi.org/10.4213/rm940

Использование Общероссийского математического портала Math-Net.Ru подразумевает, что вы прочитали и согласны с пользовательским соглашением

http://www.mathnet.ru/rus/agreement

Параметры загрузки:

IP : 54.166.219.16

26 апреля 2023 г., 14:50:27 


\title{
ОПРЕДЕЛЕНИЕ ОТНОСИТЕЛЬНОЙ ГРУБОСТИ И ДВУПАРАМЕТРИЧЕСКОЕ СЕМЕЙСТВО ФАЗОВЫХ ПОРТРЕТОВ В ДИНАМИКЕ ТВЕРДОГО ТЕЛА
}

\author{
М. В. ШАмолин
}

1. Определение относительной грубости. Классическое определение грубости [1], а также определение, данное в работе [2], оперируют с двумя объектами, а именно, с классами динамических систем и с пространством деформаций систем со своей топологией. В основе же понятия грубости лежит понятие топологической эквивалентности. Определение, данное Андроновым и Понтрягиным [1], наряду с близостью в некоторой топологии рассматриваемой системы и ее деформации, требует близость к тождественному гомеоморфизма, через который осуществляется топологическая эквивалентность. Определение же, данное Пейксото [2], не требует указанной близости. Согласно данным определениям в [1], [2], [3] изучались маломерные грубые системы, а в [4] - теория систем Аносова.

В силу классических определений структурной устойчивости, в [5], [6] обсуждаются критерии последней как для линейных неавтономных систем, так и для классов нелинейных систем. Признаки структурной устойчивости для маломерных систем формулируются в качестве гипотез Смейла на большие размерности [7].

Несколько появившихся видоизмененных определений грубости [8] имеют одно сходство: деформация рассматриваемых динамических систем на некотором многообразии $M^{n}$ берется во всем пространстве гладких векторных полей $\chi\left(C^{r}\right)$ в $C^{r}$-топологии (обычно $\left.r=1\right)$.

Рассмотрим векторные поля, деформируемые не над всем классом полей, а лишь над некоторым подклассом $\chi(\mathscr{B})$, определенным с помощью класса функций $\mathscr{B} \subset C^{r}$.

ОпредЕлЕниЕ 1. Векторное поле $\boldsymbol{v}$ называется относителњно структурно устойчивым (относительно грубым), если для любой окрестности $Г$ гомеоморфизма $\mathbf{1}_{M^{n}}$ в пространстве всех гомеоморфизмов с $C^{0}$-топологией имеется такая окрестность $\mathscr{U} \subset \chi(\mathscr{B})$ рассматриваемого векторного поля $\boldsymbol{v}$, что последнее эквивалентно любому векторному полю из $\mathscr{U} \subset \chi(\mathscr{B})$ посредством некоторого гомеоморфизма из $\Gamma$.

Заметим, что близость векторных полей понимается в $C^{1}$-топологии, а близость гомеоморфизма - в $C^{0}$-топологии. При этом речь идет не о сопряжении, а об эквивалентности.

Заметим также, что пока в вышеприведенном определении важны: 1) достаточная малость гомеоморфизма, осуществляющего эквивалентность; 2) $C^{1}$-топология в пространстве векторных полей.

2. Относительная негрубость различных степеней. Подобно тому, как дается определение первой степени негрубости, можно определить поля первой степени относительной негрубости.

ОПРЕДЕЛЕнИЕ 2. Векторное поле $\boldsymbol{v}$ называется векторным полем первой степени относительной негрубости, если оно не является относительно грубым векторным полем и если для любой окрестности $Г$ гомеоморфизма $\mathbf{1}_{M}^{n}$ в пространстве всех гомеоморфизмов с $C^{0}$-топологией имеется такая окрестность $\mathscr{U} \subset \chi(\mathscr{B})$ векторного поля $\boldsymbol{v}$, что поле $\boldsymbol{v}$ топологически эквивалентно любому полю из $\mathscr{U} \subset \chi(\mathscr{B})$, не являющемуся относительно грубым, посредством некоторого гомеоморфизма из $\Gamma$.

Близость векторных полей здесь понимается в $C^{3}$-топологии.

Аналогичным образом, с использованием $C^{2 n+1}$-топологии, определяются поля $n$-й степени относительной негрубости.

3. Динамика твердого тела, взаимодействующего со средой. В динамической системе, возникающей в динамике твердого тела, взаимодействующего со средой [9], [10], выделяется независимая подсистема второго порядка:

$$
\begin{aligned}
\alpha^{\prime} & =\omega+\frac{\sigma}{I} \mathscr{F}(\alpha) \cos \alpha+\sigma \omega^{2} \sin \alpha+\frac{s(\alpha)}{m} \sin \alpha, \\
\omega^{\prime} & =-\frac{1}{I} \mathscr{F}(\alpha)-\omega \Psi(\alpha, \omega), \\
v^{\prime} & =v \Psi(\alpha, \omega),
\end{aligned}
$$


где $\Psi(\alpha, \omega)=-\sigma \omega^{2} \cos \alpha+\frac{\alpha}{T} \mathscr{F}(\alpha) \sin \alpha-\frac{s(\alpha)}{m} \cos \alpha$. Здесь $\sigma, m, I-$ физические постоянные.

Фазовые траектории системы (1)-(2) в пространстве $\mathbb{S}^{1}\{\alpha \bmod 2 \pi\} \times \mathbb{R}^{1}\{\omega\} \times \mathbb{R}_{+}^{1}\{v\}$ лежат на поверхностях, являющихся, вообще говоря, двумерными цилиндрами. Таким образом, строение фазовой плоскости $\mathbb{R}^{2}\{\alpha, \omega\}$ системы (1) полностью определяет расслоение фазового пространства. Из относительной грубости системы (1), будет следовать относительная грубость системы (1)-(2).

В систему (1)-(2) наряду с физическими параметрами входит бесконечное число параметров, поскольку в ней имеются функции $\mathscr{F}$ и $s$. Информация о последних носит качественный характер, поскольку используется экспериментальная информация.

Следуя определению 1 , можно выделить класс функций $\mathscr{B}$ [9], [10], с помощью которого определяется пространство векторных полей $\chi(\mathscr{B})$ системы (1).

Заметим, что топология в только что определенном пространстве $\chi(\mathscr{B})$ векторных полей системы (1) индуцируется из пространства $\chi\left(C^{0}\right)$, несмотря на замечание 2) к определению 1.

В [9], [10] указано на наличие семейства фазовых портретов системы (1), в которой имеются два безмерных параметра $\left(\mu_{1}\right.$ и $\left.\mu_{2}\right)$; в пространстве последних $J^{2}=\left\{\left(\mu_{1}, \mu_{2}\right) \in \mathbb{R}^{2}: \mu_{1}>0\right.$, $\left.0<\mu_{2}<2\right\}$, рассмотрим подобласть (при $\alpha=0$ доопределим по непрерывности)

$$
\left\{\left(\mu_{1}, \mu_{2}\right) \in J^{2}: \forall \alpha \in\left(-\frac{\pi}{2}, \frac{\pi}{2}\right): \frac{s(\alpha)}{m \cos \alpha}>\frac{\sigma \mathscr{F}(\alpha)}{I \sin \alpha}\right\} .
$$

Теорема. Пространство $\chi(\mathscr{B})$, отвечающее системе вида (1), разбивается на счетное непересекающееся обвединение

$$
\chi(\mathscr{B})=\chi\left(\mathscr{B}_{1}\right) \amalg \chi\left(\mathscr{C}_{1}\right) \amalg \chi\left(\mathscr{B}_{2}\right) \amalg \chi\left(\mathscr{C}_{2}\right) \amalg \ldots,
$$

обладающее следующими свойствами:

а) система вида (1) (абсолютно) груба в пространствах $\chi\left(\mathscr{B}_{1}\right)$ для любого $i \in \mathbb{N}$,

б) система (1) является системой первой степени относительной негрубости относительно пространств $\chi\left(\mathscr{C}_{1}\right)$ для любого $i \in \mathbb{N}$,

в) мнозсества $\chi\left(\mathscr{C}_{1}\right)$ имеют меру нуль в пространстве $\chi(\mathscr{B})$,

г) множества $\chi\left(\mathscr{B}_{1}\right)$ имеют конечную меру в пространстве $\chi(\mathscr{B})$.

Более того, гомеоморфизм, осуществляющий эквивалентность систем, взятых из пространства $\chi\left(\mathscr{B}_{1}\right)$, может и не быть достаточно близок к тождественному.

Таким образом, имеем двупараметрическое семейство фазовых портретов, в котором при переходе от одного топологического типа портрета к другому мы вынуждены иметь дело с вырожденными перестройками. Причем рассматриваемые подмножества в пространстве $J^{2}$, с помощью которых определяются подклассы полей $\chi\left(\mathscr{B}_{i}\right), \chi\left(\mathscr{C}_{i}\right)$ накапливаются возле точки $(0,0) \in$ $J^{2}\left\{\mu_{1}, \mu_{2}\right\}$.

\section{СПИСОК ЛИТЕРАТУРЫ}

[1] Андронов А. А., Понтрягин Л. С. // ДАН СССР. 1937. Т. 14. № 5. С. 247-250. [2] Peixoto M. // Ann. Math. 1959. V. 69. № 2. P. 199-222. [3] Peixoto M. // Topology. 1962. V. 1. № 2. Р. 101-120. [4] Аносов Д. В. // Труды МИАН. 1967. Т. 90. [5] Плисс В. А. Интегральные множества периодических систем дифференциальных уравнений. М.: Наука, 1967. [6] Плисс В.А.// Дифференц. уравнения. 1980. Т. 16. № 10. С. 1891-1892. [7] Смейл С. // УМН. 1970. Т. 25. № 1. С. 113-185. [8] Нитецки З. Введение в дифференциальную динамику. М.: Мир, 1975. [9] Шамолин М. В. Качественный анализ модельной задачи о движении тела в среде со струйным обтеканием. Дис. ... канд. физ.-матем. наук. М.: МГУ, 1991. [10] Шамолин М.В. // ДАН. 1994. Т. 337. № 5. С. 588-591.

Московский государственный

Принято редколлегией университет им. М.В. Ломоносова 